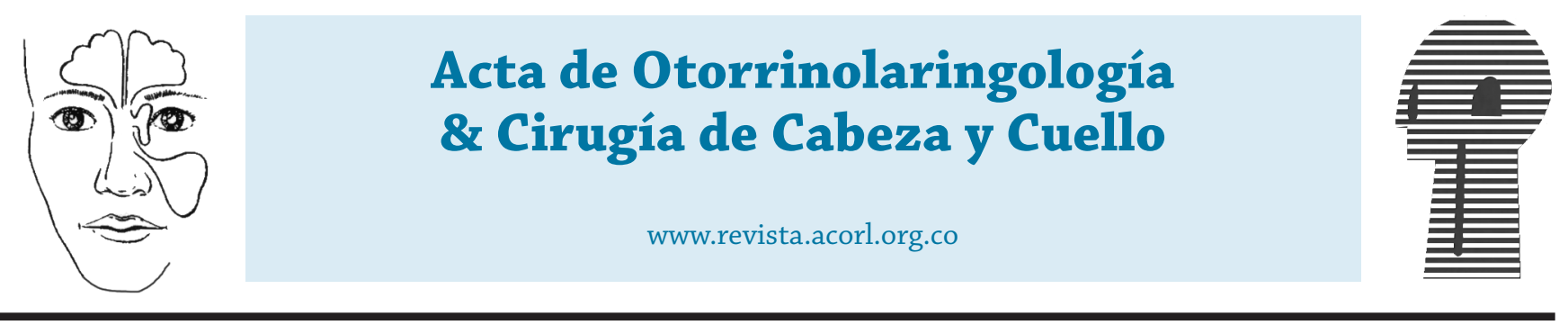

Reporte de caso

\title{
Concha bullosa gigante: reporte de un caso.
}

\section{Giant concha bullosa: a case report.}

\author{
Gabriel Eduardo Saffon Botero*
}

* Médico otorrinolaringólogo, Universidad de Caldas. Jefe del Servicio de Otorrinolaringología, Clínica Santillana. Manizales, Colombia.

Forma de citar: Saffon-Botero GE. Concha bullosa gigante: reporte de un caso. Acta otorrinolaringol. cir. cabeza cuello. 2019;47(4):238-240 Doi: 10.37076/acorlv47i4.427

\section{INFORMACIÓN DEL ARTÍCULO}

Historia del artículo:

Recibido: 25 de Abril de 2019

Revisado: 24 de Junio de 2019

Aceptado: 14 de Noviembre de 2019

Palabras clave (DeCS):

Procedimientos Quírurgicos Nasales, Endoscopía, Obstrucción Nasal, Senos Paranasales.

\section{RESUMEN}

La concha bullosa es una variante anatómica del cornete medio nasal. Se reporta un caso de una mujer de 21 años con síntomas de obstrucción nasal severa de ambas fosas nasales. Al examen físico se aprecia gran masa que ocupa la fosa nasal izquierda y una severa desviación septal a la derecha. La tomografía computarizada muestra una concha bullosa gigante izquierda, con neumatización del cornete superior izquierdo y una desviación y adelgazamiento septal severos hacia la fosa nasal derecha. Se realiza cirugía endoscópica con resección de la concha bullosa y corrección de la neumatización del cornete superior, antrostomía maxilar derecha y se lleva a cabo septoplastia como reto quirúrgico, por su grado de dificultad y por la posibilidad de causar complicaciones como la perforación septal. 


\section{ABSTRACT}

Key words (MeSH):

Nasal Surgical Procedures, Endoscopy, Nasal Obstruction, Paranasal Sinuses.
Concha bullosa is an anatomical variant of the middle nasal conchae. This is a clinical report of a case of a 21 year old woman with severe bilateral nasal fossa obstruction symptoms. During the examination, a large mass was occupying the left nasal fossa. There was also a severe deviation to the right. CT scans showed a giant left Concha bullosa, pneumatization of the superior left Concha with septal thinning and deviation towards the right nasal fossa. Endoscopic surgery is performed including resection of the Concha bullosa, correction of the pneumatization of the superior turbinate, and maxillary antrostomy, in addition, septoplasty is performed as a surgical challenge, due to its degree of difficulty and the possibility of causing complications such as septal perforation.

\section{Introducción}

La neumatización de la concha bullosa del cornete medio es la variante anatómica más común de esta estructura, es descrita con una frecuencia del $14 \%$ al $53 \%$ de los hallazgos tomográficos de los senos paranasales (1).

Sin embargo, la mayoría de las veces los pacientes son asintomáticos, se considera como una variante normal y no requiere de tratamiento, a no ser que cause obstrucción nasal o esté acompañada de otras patologías nasales y paranasales (2).

\section{Hallazgos clínicos}

Paciente femenina de 21 años que se presenta con cuadro de obstrucción nasal bilateral severa progresiva y cefalea. Al realizarse rinoscopia anterior se aprecia una masa que ocupa toda la fosa nasal izquierda, regular, rojiza rosada y dura que pasa la línea media hacia el lado derecho e impacta el septum nasal contra la pared lateral de la fosa nasal derecha, con una pirámide nasal central y una formación nasal externa central. La paciente no presenta enfermedades asociadas, ni síntomas diferentes a su obstrucción nasal. Según el interrogatorio hecho a la paciente y a su madre, no hay antecedentes de trauma nasal, ni procesos infecciosos a repetición de la vía aérea alta, ni alergias ni asma bronquial.

La tomografía nasal computarizada de los senos paranasales mostró una concha bullosa de gran tamaño que oprime la pared lateral nasal izquierda, pasa la línea media y comprime el septum nasal contra la pared lateral nasal derecha, causando una deflexión septal severa a la derecha con una estructura septal ósea adelgazada.

Se realiza plan quirúrgico y se interviene endoscópicamente resecando la lamela medial y lateral conservando la inserción del cornete medio como un remanente de referencia, se interviene endoscópicamente el cornete superior de igual lado y antrostomía media derecha. Se lleva a cabo septoplastia nasal, la cual era un reto quirúrgico por el riesgo de causar una perforación septal o una deformidad nasal externa estética, o continuar con una desviación obstructiva o todos los anteriores riesgos. Luego se procede al abordaje combinado intranasal y al abordaje de rinoplastia externa con una técnica de septoplastia extracorpórea, se conservaron ambos mucopericondrios y se reposicionaron las porciones de septum osteocartilaginoso resecadas en posición central, después se colocaron laminas paraseptales de acetato.

Los síntomas obstructivos y cefalea mejoraron dramáticamente después de la intervención y se realizaron controles endoscópicos con retiro de láminas paraseptales a los 8 días y retiro de costras, se lograron unas fosas nasales permeables con un septum funcional.

\section{Discusión}

Se describe el caso de una persona joven sin riesgos aparentes clínicos ni traumáticos, ni infecciosos o alérgicos para explicar su patología. La cocha bullosa es la variante más frecuente encontrada en el cornete medio. Fue descrita por Santorini desde el año 1793 y fue el anatomista Emil Zuckerkandl quien le dio el nombre de "concha bullosa" (3).

La detección de esta patología aumentó hasta un 53 $\%$ con el advenimiento de la tomografía computarizada y se describe como la causante de síntomas en un $15 \%$ de los pacientes que la padecen (4,5). En 1991 Bolger y colaboradores, encontraron neumatización de la lamela vertical del cornete medio en un 46,2 \% de los casos y del segmento bulloso en un $31,2 \%$, y una combinación de neumatización tanto de la lamela como del bulbo en un $15,7 \%$ de los casos (2).

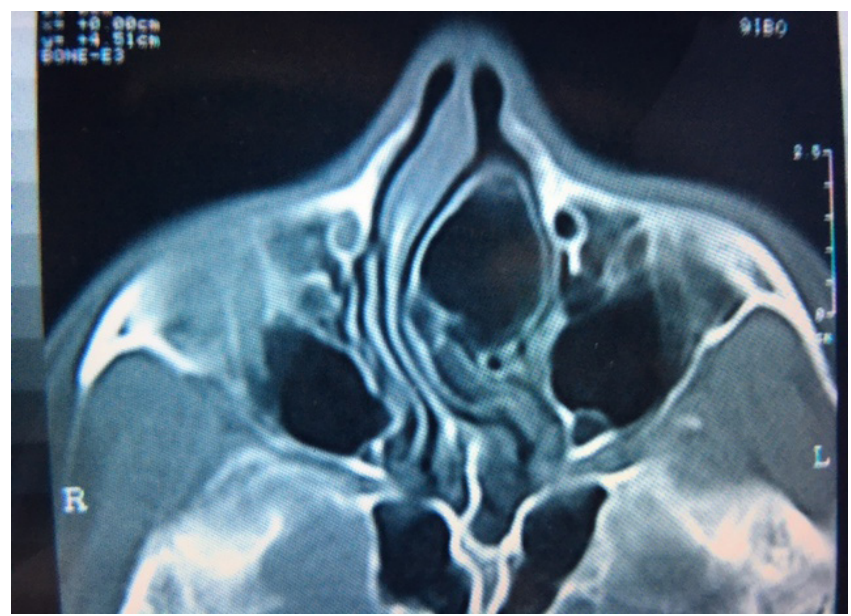




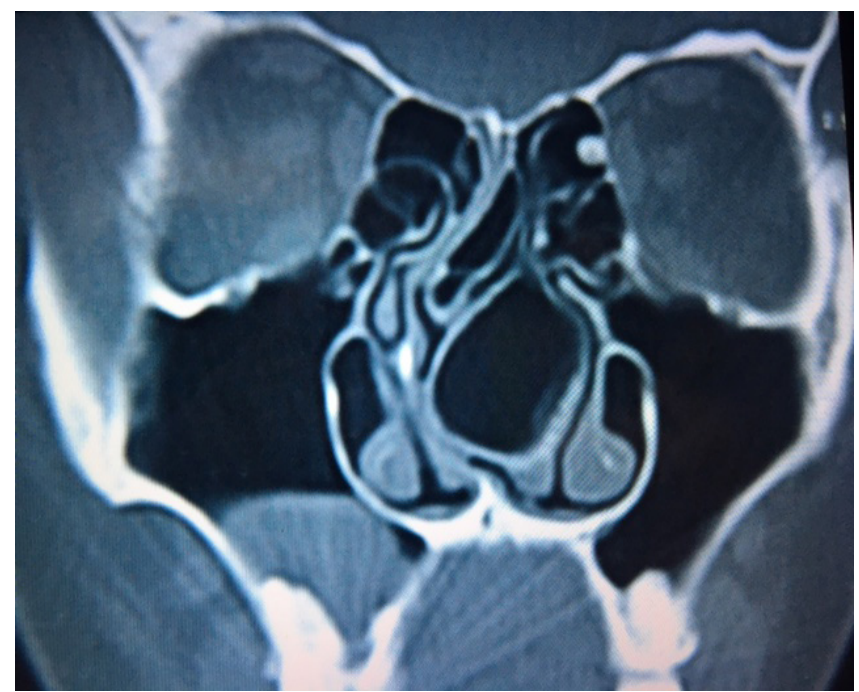

En el humano los precursores de los cornetes medios se identifican a las 8 a 10 semanas del desarrollo fetal y crecen desde la pared lateral, allí se forman 6 primordios denominados etmoidoturbinales, no todos se forman como cornetes. El primero es el proceso uncinado; el segundo, la bulla etmoidalis; el tercero, el cornete medio; y el cuarto, el cornete superior. El cornete inferior es derivado embriológicamente de una estructura diferente denominada maxiloturbinal. La neumatización del cornete medio y superior sucede a partir de estructuras óseas del complejo osteometal. El proceso de neumatización de la células etmoidales y senos es considerado un crecimiento de la mucosa del ectodermo Scheneideriano $(1,3)$.

No existe un consenso que explique el crecimiento bulloso del cornete medio y no hay una relación causal con respecto a que se presente más en pacientes alérgicos o con cuadros infecciosos en la infancia o inducidos por una desviación septal tratando el cornete medio de conservar el flujo laminar y, por ende, su crecimiento llenando el espacio $(6,7,8)$.
No es común la presentación de una concha bullosa gigante como este caso (9). Es de anotarse la buena conformación de la nariz externamente sin laterorrinias, que no se correlaciona con un trauma nasal que explique una desviación septal tan severa, se le relaciona más con la teoría del crecimiento del cornete medio bulloso per se, que empuja y se expande hacia la pared medial lateral e inferior, como en este caso en particular, queda la pregunta de cuáles son los factores que influyen el crecimiento óseo de dicho cornete.

\section{Conflicto de intereses}

El autor declara no tener conflicto de intereses.

\section{REFERENCIAS}

1. Braun H, Stammberger H. Pneumatization of turbinates. Laryngoscope. 2003;113(4):668-72.

2. Bolger WE, Butzin CA, Parsons DS. Paranasal sinus bony anatomic variation and mucosal abnormalities: $\mathrm{CT}$ analysis for endoscopy sinus surgery. Laryngoscope. 1991;101(1 Pt 1):56-64.

3. Unlu HH, Akyar S, Caylan R, et al. Concha bullosa. J Otolaryngol. 1994;23(1):23-7

4. Belli E, Rendine G, Mazzone N. Concha bullosa: endoscopic treatment. J Craniofac Surg. 2009;20(4):1165-8.

5. Basic N, Basic V, Jelic M, et al. Pneumatization of the middle nasal turbinate: a CT study. Lijec Vjesn. 1998;120(7-8):200-1

6. Pérez Piñas I, Sabaté J, Carmona A, et al. Anatomical variations in the human paranasal sinus region studied by CT. J Anat. 2000,197(Pt 2):221-7.

7. Zinreich SJ, Mattox DE, Kennedy DW, et al. Concha bullosa: CT evaluation. J Comput Assist Tomogr. 1988, 12(5):778-84.

8. Uygur K, Tuz M, Dogru H. The correlation between septal deviation and concha bullosa. Otolaryngol Head Neck Surg. 2003;129(1);33-6.

9. Ozgursoy OB, Kucuk B. Giant concha bullosa containing another concha bullosa inside: unique anatomic variation of the middle turbinate. Rhinology. 2007;45(3):248-50. 